\title{
ON THE ANALYSIS OF STUDIES OF CHOICE
}

\author{
Eamonn Mullins, Christian C. Agunwamba, and \\ Anthony J. Donohoe
}

TRINITY COLLEGE DUBLIN AND UNIVERSITY OF NIGERIA

\begin{abstract}
In a review of 103 sets of data from 23 different studies of choice, Baum (1979) concluded that whereas undermatching was most commonly observed for responses, the time measure generally conformed to the matching relation. A reexamination of the evidence presented by Baum concludes that undermatching is the most commonly observed finding for both measures. Use of the coefficient of determination by both Baum (1979) and de Villiers (1977) for assessing when matching occurs is criticized on statistical grounds. An alternative to the loss-in-predictability criterion used by Baum (1979) is proposed. This alternative statistic has a simple operational meaning and is related to the usual F-ratio test. It can therefore be used as a formal test of the hypothesis that matching occurs. Baum (1979) also suggests that slope values of between .90 and 1.11 can be considered good approximations to matching. It is argued that the establishment of a fixed interval as a criterion for determining when matching occurs, is inappropriate. A confidence interval based on the data from any given experiment is suggested as a more useful method of assessment.

Key words: matching relationship, undermatching, coefficient of determination, statistical tests, confidence intervals, concurrent variable-interval schedules
\end{abstract}

This paper is concerned with models for data arising out of experiments with concurrent variable-interval schedules. The systematic variation in such data is considered to be described by the relationship

where

$$
\log \frac{B_{1}}{B_{2}}=a \log \frac{r_{1}}{r_{2}}+\log b
$$

$B_{1} / B_{2}$ is the ratio of responses or time spent at two alternatives; $r_{1} / r_{2}$ is the ratio of the reinforcements; $a, b$ are the model parameters.

Baum (1979) examined a large body of data to establish what values of $a$ occur in practice. Undermatching is defined as occurring when $a$ is less than 1.0, matching when $a$ equals 1.0 , and overmatching when $a$ is greater than 1.0.

This paper has three sections. The first reexamines the results presented by Baum and rejects his conclusion that the time measure generally conformed to the matching relationship. The second section examines one of the statistical techniques used by Baum to assess the fits of the models. This method, despite its apparently attractive properties, is not recom-

We thank Leo Baker of the Psychology Department, Trinity College, Dublin for critical comments on an earlier draft and for useful suggestions. Reprints may be obtained from Eamonn Mullins, Department of Statistics, Trinity College, Dublin 2, Ireland. mended for future analyses because, as will be shown, the results it produces cannot be easily interpreted. An alternative method is described. In the last section it is argued that the establishment of an interval such as that proposed by Baum, within which values of $a$ can be considered as good approximations to matching, is not appropriate.

\section{THE RELATIONSHIP FOR TIME MEASURES}

Baum presented a diagram (Figure 3, p. 275) that shows a modal value of 1.0 for the slopes from experiments that reported time measures. On this basis he concluded that "the measure of time generally conformed to the matching relation but that one or more common factors make for undermatching" (p. 275). Table 1 shows the summary statistics calculated by Baum. It is at once noticeable that apart from the mode all the other summary statistics are less than unity for the time measure. The conformity to matching is therefore in some doubt.

The class intervals used for the frequency distribution that gave a mode of 1.0 were rather wide (equal logarithmic intervals, .20 in width). The data are presented in Table 2, which shows a frequency distribution whose 
Table 1

Summary statistics presented by Baum (1979) for estimated slopes for both responses and time.

\begin{tabular}{lcc}
\hline & Responses & Time \\
\hline \hline Mode & .79 & 1.0 \\
Median & .83 & .92 \\
Geometric Mean & .82 & .89 \\
\hline
\end{tabular}

class intervals are .10 units wide. A logarithmic scale was not used, since the arithmetic scale is the natural one for slope values obtained from Equation 1. Table 2 shows the modal classes for both the response and the time measures to be below 1.0. For responses the modal class interval is .75-.85, whereas for time it is .85-.95. The cumulative relative frequency column further supports the view that the time slopes do not correspond to matching, by showing that more than $60 \%$ of the studies in which time was measured gave slopes of .95 or less.

Table 3 classifies the slopes according to size and type of response measure. This table shows that two thirds of the observed slope values for the time measure are less than 1.0. This departure from symmetry with only 17 values greater than 1.0 is statistically significant $(P<.02)$. The tendency towards undermatching is, however, even stronger for the response measure with approximately $85 \%$ of the values being less than 1.0.

Table 2

Frequency distributions and cumulative relative frequency distributions of slopes for responses and time measures. Data taken from Baum (1979).

\begin{tabular}{|c|c|c|c|c|}
\hline \multirow{2}{*}{$\begin{array}{c}\text { Class Intervals } \\
\text { for Slopes } \\
>\leq\end{array}$} & \multicolumn{2}{|c|}{ Frequencies } & \multicolumn{2}{|c|}{$\begin{array}{c}\text { Cumulative } \\
\text { Relative } \\
\text { Frequencies }\end{array}$} \\
\hline & Responses & Time & Responses & Time \\
\hline $0.35-0.45$ & 0 & 1 & .00 & .02 \\
\hline $0.45-0.55$ & 0 & $\mathbf{0}$ & .00 & .02 \\
\hline $0.55-0.65$ & 5 & 2 & .10 & .06 \\
\hline $0.65-0.75$ & 10 & 9 & .28 & .24 \\
\hline $0.75-0.85$ & 16 & 6 & .60 & .35 \\
\hline $0.85-0.95$ & 11 & 13 & .81 & .61 \\
\hline $0.95-1.05$ & 7 & 8 & .94 & .77 \\
\hline $1.05-1.15$ & 2 & 10 & .98 & .96 \\
\hline $1.15 \cdot 1.25$ & $\mathbf{0}$ & $\mathbf{0}$ & .98 & .96 \\
\hline $1.25-1.35$ & 1 & 1 & 1.00 & .98 \\
\hline $1.35-1.45$ & $\mathbf{0}$ & $\mathbf{0}$ & 1.00 & .98 \\
\hline $1.45-1.55$ & 0 & 1 & 1.00 & 1.00 \\
\hline Total & 52 & 51 & & \\
\hline
\end{tabular}

Table 3

Slope estimates classified by size and type of response measure.

\begin{tabular}{lcc}
\hline & $a \geq 1.0$ & $a<1.0$ \\
\hline \hline Responses & 8 & 44 \\
Time & 17 & 34 \\
\hline
\end{tabular}

Since the modal class for the time slopes is $.85-.95$ and two thirds of the studies gave slopes of less than 1.0, undermatching must be considered to be the most commonly observed behavior. Baum's conclusion that "the measure of time generally conformed to the matching relation" must therefore be rejected.

In this paper we follow Baum and the authors he reviewed in assuming that the variable log reinforcement ratio can be considered fixed; this is implicit in the use of regression analysis. Davison and McCarthy (1981) have, however, pointed out that "the assessment of the adequacy of the matching law by least squares linear regression leads to an underestimation of reinforcement sensitivity if there is variance in the independent variable, log reinforcement ratio." We believe that the approach discussed in their paper should be explored.

\section{EVALUATING THE FITS OF THE MODELS}

For the purpose of the previous analysis, it has been assumed that it is appropriate to fit linear models to the transformed data. Baum rightly points out the need to assess the appropriateness of the functional form of the model. This must be done in all cases: fitting a straight line where a curve would be more appropriate can still result in a high value of $R^{2}$, the coefficient of determination. It is, therefore, not sufficient to rely on high $\mathbf{R}^{2}$ values when assessing the goodness-of-fit of the model. Various methods have been proposed for the assessment of functional form and the other assumptions usually made in regression analyses (see, e.g., Draper \& Smith, 1966). These techniques, which involve graphical analysis of the deviations about the fitted line, fall under the general title of residual analysis. They will, in general, be more informative than Baum's procedure of comparing the least-squares slope with that obtained by a nonparametric method. 
As a single descriptive measure of the predictive power of a regression model both Baum (1979) and de Villiers (1977) use the coefficient of determination, $\mathbf{R}^{2}$. This is, in general, a very useful statistic having, unlike many statistics, a simple operational meaning: it measures the proportion of the total variation in the response variable that can be accounted for by the variation in the predictor variable(s). Unfortunately, in the special case considered by both these authors, viz., the slope is a priori constrained to equal 1.0 , this statistic loses this simple operational meaning.

The coefficient of determination is derived from the following decomposition of the total variation in the response variable $\left(Y_{i}\right)$ :

$$
\begin{aligned}
& \sum_{i}\left(Y_{i}-\bar{Y}\right)^{2}= \sum_{i}\left(Y_{i}-\hat{Y}_{i}\right)^{2}+\sum_{i}\left(\hat{Y}_{i}-\bar{Y}\right)^{2} \\
&+2 \underset{i}{\operatorname{SSR}}\left(Y_{i}-\hat{Y}_{i}\right)\left(\hat{Y}_{i}-\bar{Y}\right) \\
& \text { SSCP }
\end{aligned}
$$

where

$i \quad$ labels the data points from 1 to $N$

$Y_{i} \quad$ is the $i^{\text {th }}$ observation of the response variable

$Y_{i} \quad$ is the $i^{\text {th }}$ fitted value; i.e., the point on the regression line corresponding to $Y_{i}$

SSTO (total sum of squares) measures the variation in $Y$

SSR (regression sum of, squares) measures that part of the variation in $Y$ that is predicted by the regression line

SSE (error sum of squares) measures that part of the $Y$ variation that can be considered as random if we regard the regression line as describing the systematic variation in $Y$

SSCP (cross-product sum of squares).

If all the parameters of the model are estimated by least squares from the data (here there are only two parameters-the slope and intercept) the cross-product term (SSCP) equals zero. Then the total variation in $Y$ decomposes into a systematic component (SSR) and a random component (SSE). Thus,

$$
\mathbf{R}^{2}=\frac{\text { SSR }}{\text { SSTO }}=1-\frac{\text { SSE }}{\text { SSTO }}
$$

$\mathbf{R}^{2}$ obviously lies between 0 and $\mathbf{l}$ as it is a proportion. Values close to 1 indicate good predictive power under the conditions for which the data were obtained, provided there is no evidence of nonlinearity.
If, however, any of the parameters are fixed a priori, the cross-product term is no longer constrained to be zero, and the simple decomposition into only regression and error terms no longer holds. $\mathbf{R}^{2}$ is not then necessarily constrained to lie in the interval zero to unity and so loses its simple interpretative property. An example of this can be seen in Table 1 of Baum (1979). Subject 334 (Baum 1973) gave an $R^{2}$ value of -1.06 ; obviously it is not meaningful to account for a negative proportion of the total variation in the response variable. It is suggested, accordingly, that this measure of goodness-of-fit is inappropriate when a priori values are specified for one or more parameters in a regression model.

To assess the deviations of slope values from 1.0, Baum asks simply "how much we lose in predictability if we assume the slope to equal 1.0" (p. 276). He defined the coefficient of determination (usually denoted $\mathrm{R}^{2}$ ) to be $H$ when the slope is estimated from the data, $H^{\prime}$ when it is preset at 1.0. He then uses

$$
\text { Loss in Predictability }=\frac{H-H^{\prime}}{H}
$$

as a measure of the deviation of the observed slope from 1.0. If it were not for the difficulties with $H^{\prime}$ discussed above, this would be a very appealing index because of its simple operational interpretation. It is sufficient to point out that $H^{\prime}$ may be negative (e.g., Subject 334 referred to above) to undermine the reliability of this measure.

An alternative measure that avoids the problems associated with the use of $H^{\prime}$ is the following:

where

$$
D=\frac{\mathrm{SSE}_{2}-\mathrm{SSE}_{1}}{\mathrm{SSE}_{1}}
$$

$\mathrm{SSE}_{1}$ is the error sum of squares from the model in which the slope is estimated from the data;

$\mathrm{SSE}_{2}$ is obtained when the slope is constrained to equal 1.0.

The deviations of the data points about the regression line contain all the information about the goodness-of-fit. To assess the deviation from 1.0 of the fitted slope we simply ask "what is the fractional increase in the error sum of squares when we force the slope to equal 1.0?". The motivation for this as a descriptive statistic lies in the fact that $\mathrm{SSE}_{1}$ 
should be a measure of random variation only. This will be the case if the least-squares line accounts for the systematic variation in the data. If, however, fixing the slope to equal 1.0 is inappropriate, $\mathrm{SSE}_{2}$ will reflect not only the random variation in the data but also the bias which has been introduced by use of an inappropriate slope. Large values of $D$ suggest therefore that the true slope is not 1.0 , whereas small values of $D$ are consistent with this hypothesis.

The $D$ statistic has been proposed primarily as a descriptive measure. It can, however, be used to obtain a formal statistical test of the hypothesis that the true slope is 1.0. If the usual assumptions for a regression model, including Normality, are valid, then $(N-2) D$ is distributed as $F(1, N-2)$, where $N$ is the number of observations. This means that $(N-2) D$ is the usual $F$-ratio statistic employed to test the hypothesis that the slope is unity.

\section{AN ACCEPTANCE REGION FOR MATCHING?}

One conclusion of Baum's (1979) paper is that "statistical techniques for assessing significance of a deviation of $a$ from 1.0 suggested that values of $a$ between .90 and 1.11 can be considered good approximations to matching" (p. 269). Since this conclusion arises out of an important large scale review of recent work, it is quite likely to be accepted as a criterion for evaluating fits in future work. The possibility of having a fixed interval for slope estimates, to determine when matching has occurred, is attractive because of its simplicity. We would, however, argue against the adoption of any such fixed interval as a decision rule, on the grounds that it ignores the variability in the particular experiment being considered. This is not to say that the interval discussed by Baum is not useful. It is an interesting descriptive summary of the results of a large number of studies.

Together with the loss-in-predictability criterion, $\frac{H-H^{\prime}}{H}$ (criticized in the last section), Baum uses a nonparametric statistical test (due to Theil, 1950) to suggest this interval. We note in passing that if it can be assumed that the distribution of the deviations about the re- gression line is approximately Normal (as could certainly be done for many of the data sets in question), a parametric test such as the $t$-test or equivalently the $F$-test would be more appropriate than a nonparametric test.

Baum found that Theil's test will accept the hypothesis that the true slope equals 1.0 if the estimate lies in the interval $(.9,1.2)$. To be clear on the implication of this result, let us consider what happens in any given test. Assume that the slope estimate is .89 with an associated probability level $(p<.20)$. Such a result would be interpreted as indicating that the sample estimate is consistent with (i.e., does not reject the hypothesis of) the model parameter being 1.0. The test would, of course, also accept that the sample estimate was consistent with a true slope of anything between .89 and 1.0 or indeed of values less than .89. If there is quite a lot of random variation present then slope estimates quite far from 1.0 will still be consistent with a true slope of 1.0. Conversely, if the amount of random variation is small, slope estimates quite close to 1.0 may be inconsistent with a true slope of 1.0. Thus, to take an extreme example, if all of a large number of observations lie exactly on a straight line of, for example, slope .97 it would hardly be appropriate to decide that the "true" slope is $\mathbf{1 . 0}$. For both of these reasons it is argued that a fixed interval is inappropriate as a criterion for determining when matching occurs. In making such a decision, account should be taken of the point estimate of the slope and also of the variability in the data. This is the purpose of a statistical test.

One consequence of doing statistical tests of any sort is that, once obtained, the test result tends to receive all the attention and such other information as is in the data tends to be ignored. It is suggested, therefore, that given the present state of knowledge concerning studies of choice, confidence intervals for model parameters, where these may be obtained, are more informative than tests. Thus, in our hypothetical example, a sample estimate of .89 might give a confidence interval of .77 to 1.01. Such an interval does three things: it gives us the information that the slope estimate is consistent with a matching relationship, as would a test; it tells us further that the data are consistent with the relationship being quite far from matching; and by the explicit 
presentation of so wide an interval we are less inclined to forget that our measurements of the behavior are as yet fairly imprecise.

Our measurements can be made more precise in two ways. The number of observations for any particular experiment can be increased. More importantly, from an experimental point of view, the factors which Baum (1979) has identified as contributing to the variability in results between observers must be studied so that they can be controlled in future experiments.

\section{REFERENCES}

Baum, W. M. Time allocation and negative reinforcement. Journal of the Experimental Analysis of Be. havior, 1973, 20, 313-322.
Baum, W. M. Matching, undermatching, and overmatching in studies of choice, Journal of the Experimental Analysis of Behavior, 1979, 32, 269-281.

Davison, M., \& McCarthy, D. Undermatching and structural relations. Behavior Analysis Letters, 1981, 1, 67-72.

de Villiers, P. Choice in concurrent schedules and a quantitative formulation of the law of effect. In W. K. Honig \& J. E. R. Staddon (Eds.), Handbook of operant behavior. Englewood Cliffs, N.J.: PrenticeHall, 1977.

Draper, N. R., \& Smith, H. Applied regression analysis. New York: Wiley, 1966.

Theil, H. A rank-invariant method of linear and polynomial regression analysis. I, Proceedings Koninklijke Nederlandse Akademie van Wetenschappen, 1950, A.53, 521-525.

Received May 8, 1981

Final acceptance October 30, 1981 